\title{
Eficiencia técnica de la atención al paciente con diabetes en el primer nivel
}

\author{
Ana María Salinas-Martínez, DSP, (I) María Agustina Amaya-Alemán, MSc, (') Julio César Arteaga-García,PhD,(2) \\ Georgina Mayela Núñez-Rocha, D en M,(I) María Eugenia Garza-Elizondo, MSc.(I)
}

\begin{abstract}
Salinas-Martínez AM,Amaya-Alemán MA, Arteaga-García JC, Núñez-Rocha GM, Garza-Elizondo ME. Eficiencia técnica de la atención al paciente con diabetes en el primer nivel. Salud Publica Mex 2009;5 I:48-58.
\end{abstract}

\begin{abstract}
Resumen
Objetivo. Cuantificar en un primer nivel la eficiencia técnica de la atención al paciente con diabetes y distinguir la provisión de servicios y los resultados en salud, además de reconocer las fuentes potenciales de variación. Material y métodos. Se incluyeron insumos, actividades clínicas y resultados en salud de 47 clínicas familiares del IMSS Nuevo León. La medida de la eficiencia se realizó mediante el análisis envolvente de datos y se aplicaron modelos de regresión de Tobit. Resultados. Siete clínicas fueron eficientes en cuanto a la provisión de servicios y nueve en salud; dos coincidieron en ambos aspectos. La localización metropolitana y el total de consultas favorecieron la eficiencia en relación con la provisión de servicios, cualesquiera que fueran los atributos del paciente; y la edad del médico, la eficiencia de los resultados en salud. Conclusiones. El desempeño varió en una misma unidad y entre una y otra; algunas fueron eficientes para suministrar servicios y otras para mejorar la salud. Las fuentes de variación también difirieron. Se recomienda incluir ambos productos en el estudio de la eficiencia en diabetes en el primer nivel.
\end{abstract}

Palabras clave: eficiencia; atención primaria; diabetes; México
Salinas-Martínez AM,Amaya-Alemán MA, Arteaga-García JC, Núñez-Rocha GM, Garza-Elizondo ME. Technical efficiency in primary care for patients with diabetes.

Salud Publica Mex 2009;5 I:48-58.

\section{Abstract}

Objective. To quantify the technical efficiency of diabetes care in family practice settings, characterize the provision of services and health results, and recognize potential sources of variation. Material and Methods. We used data envelopment analysis with inputs and outputs for diabetes care from 47 family units within a social security agency in Nuevo Leon. Tobit regression models were also used. Results. Seven units were technically efficient in providing services and nine in achieving health goals. Only two achieved both outcomes. The metropolitan location and the total number of consultations favored efficiency in the provision of services regardless of patient attributes; and the age of the doctor, the efficiency of health results. Conclusions. Performance varied within and among family units; some were efficient at providing services while others at accomplishing health goals. Sources of variation also differed. It is necessary to include both outputs in the study of efficiency of diabetes care in family practice settings.

Key words: efficiency; primary care; diabetes; Mexico

(I) Unidad de Investigación Epidemiológica y en Servicios de Salud. Instituto Mexicano del Seguro Social. México.

(2) Facultad de Economía, Universidad Autónoma de Nuevo León. México. 
Es el perfil de salud de la población mexicana sobresale la diabetes como enfermedad no transmisible, constituida en epidemia creciente en todas las regiones del país. La última Encuesta Nacional de Salud y Nutrición indica que más de $9 \%$ de los adultos en México padece diabetes y la mortalidad por esa causa aumenta a un ritmo anual de 3\%. ${ }^{1}$ La atención a este padecimiento representa un reto para cualquier organización de salud, en virtud de los efectos que representan sus complicaciones en el deterioro de la calidad de vida, demanda y consumo de servicios ambulatorios y hospitalarios. La diabetes figura entre las primeras causas de consulta de medicina familiar e incapacidad en el Instituto Mexicano del Seguro Social (IMSS) ${ }^{2}$ y entre las primeras cinco de egreso hospitalario de instituciones del sistema nacional de salud (sin incluir las afecciones ginecoobstétricas y pediátricas). ${ }^{3}$ De manera adicional, cuando se ajusta por diferencias de edad, sexo y grupo étnico, el diabético incurre en gastos médicos 2.3 veces más respecto de la población general sin diabetes. ${ }^{4}$ Más aún, se ha señalado que un cuidado apropiado de atención primaria puede evitar hasta 2500 millones de dólares en costos hospitalarios. ${ }^{5}$

La medicina familiar es una especialidad médica enfocada en la atención primaria con actividad clínica y contenido propio de enseñanza e investigación ${ }^{6} \mathrm{y}$ se espera que los centros de primer nivel de atención ejerzan sus recursos en forma eficiente a través de habilidades clínicas y gerenciales. Esta premisa es aún más trascendental para el caso de las instituciones con políticas de contención de costos, como las del sector público que dependen de un presupuesto asignado y la distribución inteligente de los recursos disponibles; es por ello que tienen una gran importancia los programas de eficiencia. La función de producción en el área de la salud consiste en convertir recursos en servicios (producto intermedio) y bienestar (producto final). En el primer nivel, le corresponde al médico familiar solicitar estudios de laboratorio, cuantificar el peso y la presión arterial y revisar los pies, entre otros servicios, además de lograr el control adecuado del paciente con diabetes. En consecuencia, el estudio de la eficiencia debe distinguir procesos y resultados de salud. Sin embargo, muchos trabajos utilizan sólo productos intermedios, a pesar de que no deben soslayarse los productos finales. ${ }^{7}$ La eficiencia técnica se ha definido como la relación entre insumos (costos en forma de mano de obra, capital o equipo) y los resultados. No establece comparaciones entre tratamientos alternos ni indica si una intervención produce un mejor resultado que otra con menos recursos; señala más bien si una organización alcanza el resultado máximo posible con base en su nivel de insumos. ${ }^{8,9} \mathrm{El}$ origen de la escasa eficiencia se puede deber a variaciones regionales, ${ }^{10}$ tipo de afiliación, ${ }^{11,12}$ ámbito no lucrativo ${ }^{13,14}$ y volumen o diversidad de servicios otorgados. ${ }^{11,15}$ Asimismo, deben considerarse los atributos del médico tratante y el paciente. ${ }^{11}$ La eficiencia de la atención en diabetes se ha analizado con amplitud desde el punto de vista de costoefectividad, pero los estudios sobre eficiencia técnica en diabetes son limitados. ${ }^{16,17}$ En México, no se dispone de informes publicados de eficiencia técnica de programas de atención a pacientes con esta enfermedad, algo que debiera subsanarse puesto que el conocimiento sobre la buena utilización de los recursos, dada su escasez, es algo con lo que debe contar toda organización pública o privada. La presente investigación se llevó a cabo en Nuevo León, región con $68.5 \%$ de población afiliada al IMSS durante el año que se desarrolló el estudio., ${ }^{2,3} \mathrm{El}$ objetivo fue calcular en dicha institución la eficiencia técnica de la atención al paciente con diabetes en el primer nivel de atención y distinguir la provisión de servicios y los resultados de salud, además de reconocer las fuentes potenciales de variación.

\section{Material y métodos}

Se estudiaron 47 de 51 clínicas de medicina familiar del IMSS-Nuevo León, entre el 1 de enero y el 31 de diciembre de 2004; una unidad no se consideró porque, dada su reciente creación, no operaba en su totalidad y las restantes eran centros satélite o consultorios auxiliares. Las unidades del primer nivel no tienen gran complejidad y operan en los turnos matutino y vespertino (Anexo 1); en la figura 1 se muestra la ubicación geográfica de las clínicas estudiadas, así como los datos estatales sobre morbimortalidad por diabetes, infraestructura y productividad de los servicios de medicina familiar, en el año de referencia. El Comité Institucional de Investigación correspondiente aprobó el proyecto; se usaron códigos alfabéticos asignados de modo aleatorio a las unidades para preservar su anonimato.

Cuantificación de la eficiencia. Se realizó mediante el análisis envolvente de datos (AED), un método de los más utilizados en el estudio de la eficiencia técnica de organizaciones de salud que no requiere el uso de precios porque se basa en cantidades de insumo y no en valores monetarios. ${ }^{18,19} \mathrm{El} \mathrm{AED} \mathrm{mide} \mathrm{la} \mathrm{eficiencia} \mathrm{técnica}$ a través del cociente entre una combinación lineal de resultados y una combinación lineal de insumos que genera una puntuación de 0 a 1 ; el 0 señala el extremo de ineficiencia y el 1, la existencia de eficiencia. Lo anterior se fundamenta en el hecho de que una clínica utiliza insumos de 1 a $r$ y produce resultados de 1 a s: $u_{1} y_{1 a}+u_{2} y_{2 a}+\ldots+\ldots u_{s} y_{s a} / v_{1} x_{1 a}+v_{2} x_{2 a}+\ldots+\ldots v_{r} x_{r a^{\prime}}$ en donde 

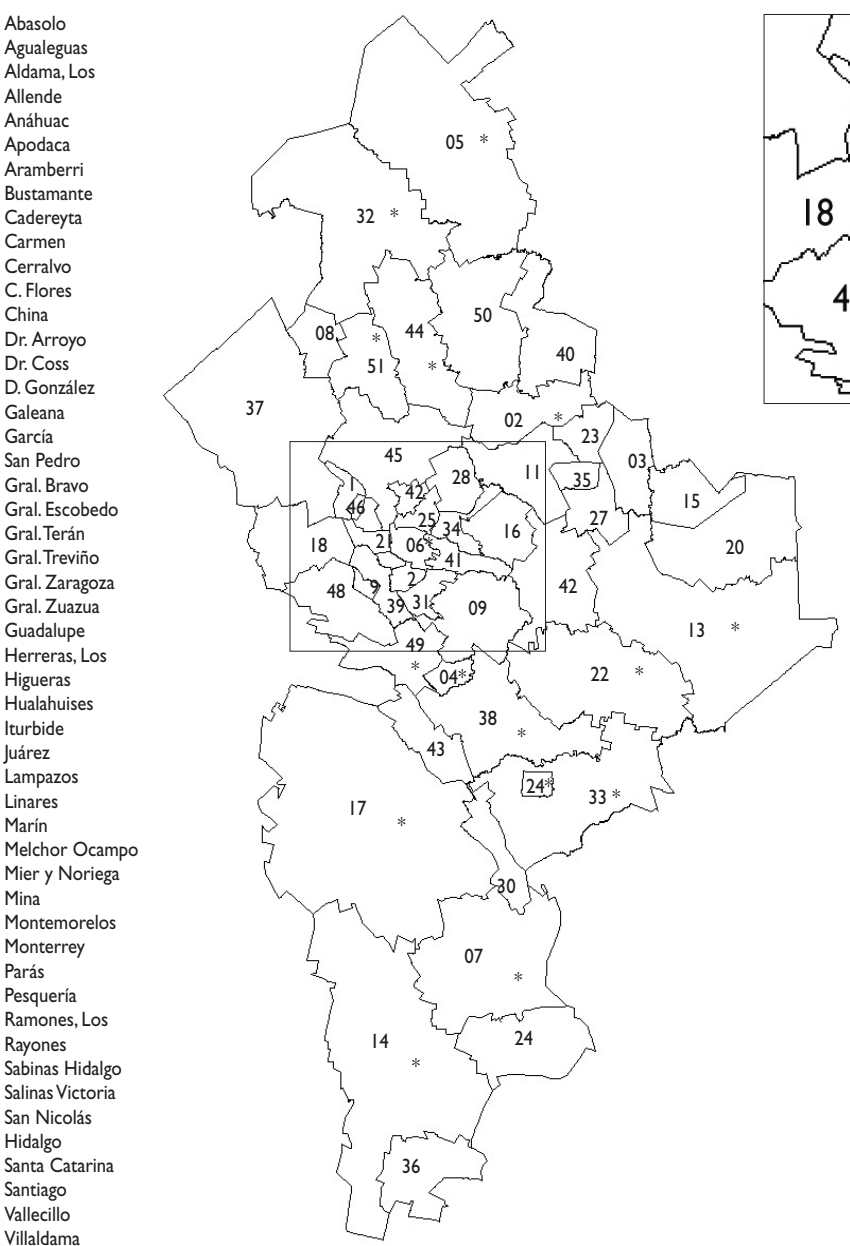

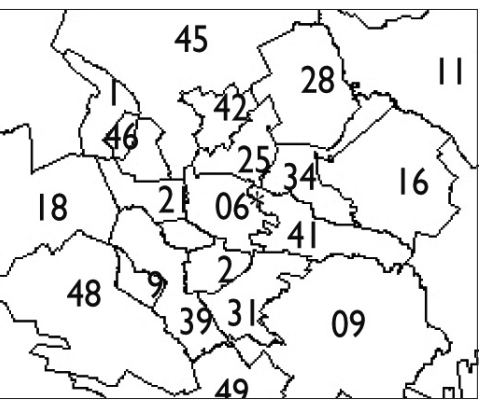

Nuevo León, 2004

\section{Morbi-mortalidad por diabetes}

Prevalencia $\geq 20$ años: 22

$9.7 \%$ ( $2^{\circ}$ lugar en el país)

- Tasa estandarizada de mortalidad: ${ }^{3}$

78.3 por 100000 (nacional 88.4), en hombres

83.9 por 100000 (nacional 90.7), en mujeres

- IMSS, ler. lugar motivo consulta en medicina familiar ${ }^{2}$

- IMSS $5^{\circ}$ lugar de egreso hospitalario (no obstetricia o pediatría) ${ }^{2}$

Infraestructura y productividad medicina familiar

- Médicos generales y familiares por I 000 habitantes: ${ }^{30}$

0.42 (nacional 0.47)

- Consultorios generales, Sistema Nacional de Salud: ${ }^{3}$ I 118

- Consultorios generales, medicina privada: ${ }^{3}$

99

- Consultas generales por 1000 habitantes: ${ }^{31}$

| 682.3 (nacional | 759)

- Egresos hospitalarios por 1000 habitantes: ${ }^{31}$

52.2 (nacional 45.4)

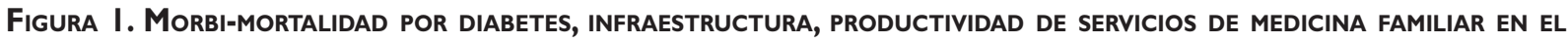
Estado de Nuevo León y ubicación geográfica de las clínicas estudiadas (*)

$u_{1}$ es el peso aplicado al resultado $1 ; y_{1 a}$ es la cantidad del resultado $1 ; v_{1}$ es el peso aplicado al insumo $1 ; \mathrm{y} x_{1 a}$ es la cantidad de recurso 1. Por consiguiente, para cada centro se calcula el conjunto de pesos que maximice su eficiencia; si un centro tiene eficiencia menor a 1, ello implica que hay otro de eficiencia 1 con el mismo conjunto de pesos. Las puntuaciones de eficiencia son relativas y se aplican a las unidades bajo estudio. ${ }^{9}$

Insumos y productos. La eficiencia de servicios incluyó como insumo la proporción de horas-hombre en atención a la diabetes, es decir, el porcentaje de tiempo de la jornada laboral que el médico ocupa en atender a pacientes con esta enfermedad. A la eficiencia de resultados en salud hay que añadir el recurso del nu- tricionista y la enfermera al servicio de los pacientes crónicos, ya que colaboran con el médico en beneficio del individuo. No se consideraron insumos materiales porque, dada la política institucional, la distribución de éstos es similar para todas las unidades del primer nivel. En cuanto a los productos, se incluyeron indicadores de provisión de servicios para la detección temprana de complicaciones, además de resultados en salud, en términos del control de glucosa, presión arterial y lípidos, ${ }^{20,21}$ y uno de percepción de calidad (cuadro I). Las fuentes de información fueron variadas; algunos datos no se encontraban disponibles en registros institucionales por lo que se obtuvieron directamente del paciente, previo consentimiento informado. Para ello 
se determinó cuántos pacientes con diabetes habría en un día en espera de consulta con base en la prevalencia para derechohabientes de $8.9 \%,{ }^{22}$ total de población usuaria y 252 días hábiles en el año. Así se identificó la meta mínima a cubrir por clínica. Para evitar que la selección se sesgara hacia un consultorio en particular, se distribuyó la cuota de un paciente por consultorio por turno: un mínimo de 10 para unidades con un solo consultorio (en total de 976). Empero, se entrevistaron y consultaron expedientes clínicos de 1101 pacientes. De manera inicial, se sorteó el día de la semana de visita a la clínica, aunque esto no resultó práctico porque ciertas clínicas concentraban las citas de control de diabetes en días específicos. No se tomó en cuenta a personas con menos de dos visitas de control durante el año anterior a la encuesta. Además, con la finalidad de disminuir la heterogeneidad y equiparar el perfil de gravedad de la enfermedad, se excluyó a pacientes con ceguera parcial o total. También, se eliminó del análisis a las personas con enfermedad renal crónica y alguna amputación en miembros inferiores (como medidas aproximadas de gravedad; $n=24$ ), además de pacientes con valores extremos en tiempo de diagnóstico, edad y escolaridad $(n=60)$. En el Anexo 2 se puede consultar la información detallada por unidad. Por otra parte, el departamento de recursos humanos proporcionó información de 803 médicos familiares fijos (de casi 900 de atención primaria) sobre edad, antigüedad y días laborados, en el año de referencia.

Especificaciones del AED. Se utilizó una escala de retorno variable y se asignó el enfoque de maximización de resultados que permite mantener constante la cantidad

\section{Cuadro I}

\section{Estadísticas descriptivas de las variables Utilizadas. NueVo León, 2004}

\begin{tabular}{ccc} 
Medición estadística \\
\cline { 2 - 4 } Media DE Mediana Percentil 25 Percentil 75 CV (\%)
\end{tabular}

Insumos

I. Porcentaje de horas-hombre-médico en atención a pacientes con diabetes*

2. Porcentaje de médicos familiares que utiliza el apoyo de nutricionistas ${ }^{\ddagger}$ $\begin{array}{lll}-- & -- & 12\end{array}$ al servicio de pacientes con afecciones crónicas ${ }^{\ddagger}$

\begin{tabular}{lll} 
ns $^{*}$ & -- & -- \\
\hline-- & -- \\
\hline- & --
\end{tabular}

12

al servicio de pacientes con afecciones crónicas ${ }^{\ddagger} \quad--\quad-\quad 200$

Productos de provisión de servicios

I. Porcentaje de pacientes con examen anual de colesterol (sólo casos sin diagnóstico previo de colesterol elevado) $\begin{array}{llllll}81 & 25 & 90 & 75 & 100 & 31\end{array}$

2. Porcentaje de pacientes con medición de presión arterial

en cada visita de control§ ${ }^{\S}$

3. Porcentaje de pacientes con medición de peso en cada visita de control ${ }^{\S}$

4. Porcentaje de pacientes con revisión anual de pies ${ }^{\ddagger}$

5. Porcentaje de pacientes con solicitud anual de fondo de ojo

\begin{tabular}{llllll}
81 & 14 & 80 & 70 & 92 & 18 \\
84 & 16 & 88 & 76 & 96 & 19 \\
30 & 19 & 28 & 14 & 50 & 63 \\
\hline 22 & 12 & 20 & 11 & 30 & 57
\end{tabular}

Productos de resultados en salud

I. Porcentaje de pacientes con glucosa en ayuno $\leq 130 \mathrm{mg} / \mathrm{dL}$ (promedio de los últimos 3 reportes de laboratorio)

$\begin{array}{llllll}24 & 13 & 22 & 17 & 33 & 53\end{array}$

2. Porcentaje de pacientes con colesterol $<200 \mathrm{mg} / \mathrm{dL}$ en el último reporte de laboratorio $\$$

$\begin{array}{llllll}43 & 21 & 46 & 30 & 57 & 49\end{array}$

3. Porcentaje de pacientes con presión arterial $<130 / 80 \mathrm{mmHg}$ al momento de la encuesta

$\begin{array}{llllll}22 & 15 & 22 & 11 & 30 & 68\end{array}$

4. Porcentaje de pacientes que considera que recibió la atención que esperaba del médico familiar la última visita de control ${ }^{\ddagger}$

$47 \quad 25$

43

26

67

\footnotetext{
* Debido a que el médico familiar no atiende sólo a pacientes con esta enfermedad, se calculó el porcentaje de horas-hombre-médico en atención a pacientes con diabetes

‡ Fuente: entrevista al paciente

$\S$ Fuente: expediente clínico del paciente encuestado

DE, desviación estándar; CV, coeficiente de variación
} 
de insumos. El cálculo de eficiencia técnica se realizó con el programa Frontier Analyst Professional de Banxia. Se usaron modelos de regresión de Tobit para analizar fuentes de variación de eficiencia después de realizar pruebas de Kolmogorov-Smirnov para la puntuación de eficiencia de provisión de servicios (K-S $z=0.99, p=0.27$ ) y para la de resultados en salud (K-S $z=1.3, p=0.16)$. El AED cataloga unidades ineficientes con un espectro amplio de valores menores a 1 , en tanto que las unidades eficientes alcanzan el 1, sin tener la opción de discriminar por arriba de dicha cifra. Por esa razón, la puntuación pertenece a una distribución censurada y no es adecuado usar modelos de regresión lineal o cuadrados mínimos. ${ }^{23}$ Se aplicó un único punto de censura igual a 1 y se calculó la $R^{2}$ entre los valores esperados y los observados. ${ }^{24}$ Después de identificar los factores relacionados con la eficiencia, se consideraron el promedio de edad, la antigüedad y los días laborados de los médicos familiares fijos. La edad y la antigüedad laboral se correlacionaron de manera notoria y significativa $(r=0.81$, $p<0.001)$ y se decidió conservar la primera después de observar resultados similares en la bondad de ajuste de los modelos. También se incluyó la localización de la unidad y el total de consultas, además del porcentaje de pacientes con escolaridad hasta primaria, promedio de edad, tiempo con el diagnóstico, tiempo de afiliación y visitas de control. El análisis de Tobit se realizó con el paquete estadístico EasyReg International. ${ }^{25}$

\section{Resultados}

En el cuadro I se muestra la estadística descriptiva de los insumos y resultados en salud; la media y la desviación estándar se proporcionan sólo para variables con distribución normal. El AED mostró siete clínicas eficientes en la provisión de servicios y nueve en resultados de salud, lo que indica que son del todo eficientes en uno o más de los indicadores analizados; el rendimiento a escala en todas ellas fue constante. Los centros restantes no fueron eficientes en ningún indicador y registraron un rendimiento creciente a escala (cuadro II). El promedio de la puntuación en la provisión de servicios fue $69.3 \%$ (IC95\% 61, 77) y en resultados de salud 74.2\% (IC95\% 67, 82); sólo dos unidades fueron eficientes en ambos productos, la unidad AE y la unidad I. De modo adicional, el AED informó sobre el porcentaje de mejoría potencial dado el nivel de recursos de la unidad. Como se puede observar en el cuadro III, todos los centros

\footnotetext{
* Banxia Efficiency Analysis Software. (Frontier Analyst 3 Professional). UK: Banxia Holding Limited, 1998-2001.
}

que operan con puntuación de eficiencia inferior a 50 podrían mejorar en $100 \%$ la actividad examen anual de pies y el resultado control de glucosa en sangre.

Origen de la eficiencia. El análisis descriptivo de las variables seleccionadas mostró un promedio de edad del médico familiar de $46 \pm 3.4$ años y antigüedad de $14.9 \pm 3.9$ años, mientras que para los días laborados en el año de referencia se obtuvo una cifra de $243.9 \pm 60$.2. En lo que respecta a la población encuestada, el promedio de edad correspondió a 59.4 \pm 3.6 años; el tiempo con el diagnóstico fue de 9.9 2.6 años; el tiempo de afiliación de $24.2 \pm 6$ años; y las visitas de control durante el año de referencia de $9.54 \pm 2.29$. El porcentaje promedio con escolaridad hasta primaria fue de $79.6 \pm 13.8$. Se analizaron dos modelos de Tobit para las puntuaciones de eficiencia en provisión de servicios y resultados en salud, uno con ubicación de la unidad y otro con total de consultas (cuadro IV), toda vez que ambas variables estuvieron estrechamente relacionadas entre sí $(r=0.79, p<0.001)$. Los modelos para provisión de servicios mostraron diferencia significativa entre el modelo propuesto con las variables explicativas y aquél que contenía sólo la constante; el modelo con zona de ubicación registró 83\% de unidades con valor esperado dentro de la desviación estándar del valor observado y $79 \%$ aquél con total de consultas. Por su parte, los modelos de eficiencia en resultados de salud fueron de 77 y $79 \%$, respectivamente. En el cuadro IV se puede observar que la localización en la zona metropolitana y el mayor número de consultas favorecieron la eficiencia en el suministro de servicios, ya que presentaron coeficientes con signo positivo $(p \leq 0.01)$. Ninguno de estos dos factores influyó en grado significativo en la eficiencia de resultados en salud. En cuanto a las características del médico, el signo positivo del coeficiente de tiempo laborado fue consistente, pero no la relevancia estadística, con predominio de la ausencia de efecto. La edad no favoreció que las unidades fueran eficientes en proveer servicios, pero sí para obtener resultados en salud ( $p \leq 0.01)$. La participación de las variables inherentes al paciente fue mixta. Se observó que a mayor edad o tiempo con el diagnóstico, mayor eficiencia en provisión de servicios, aunque menor eficiencia de resultados en salud; lo contrario sucedió con el tiempo de afiliación.

\section{Discusión}

La presente investigación calculó, mediante un abordaje de corte transversal, la eficiencia técnica de unidades médicas familiares en relación con la atención al paciente con diabetes. El promedio de eficiencia en provisión de servicios fue de $69 \%$ y en salud de $74 \%$. 
Cuadro II

Puntuaciones de eficiencia de PROVISión de SERVICIOS Y Resultados EN SALUd unidAdes de SALUd del PRIMER NIVEl de ATENCIÓN. NueVo León, 2004

$\begin{array}{cc}\text { Puntuación de eficiencia } \\ \text { Unidad médica } & \text { en provisión de servicios (\%) }\end{array}$

Puntuación de eficiencia de resultados en salud (\%)

\author{
Diferencia \\ de puntuaciones*
}

Lugar que ocupa la unidad con base en servicios
Lugar que ocupa la unidad con base en salud

\begin{tabular}{|c|c|c|c|c|c|}
\hline $\mathrm{AH}$ & $<30$ & 45 & - & 38 & 33 \\
\hline$A R$ & $<30$ & 65.6 & - & 38 & 26 \\
\hline $\mathrm{H}$ & $<30$ & 97 & - & 38 & 3 \\
\hline Q & $<30$ & 100 & - & 38 & I \\
\hline $\mathrm{Al}$ & 37 & 51.3 & - & 37 & 31 \\
\hline G & 37.9 & 100 & - & 36 & I \\
\hline $\mathrm{L}$ & 40 & 100 & - & 35 & I \\
\hline $\mathrm{J}$ & 40.5 & $<30$ & + & 34 & 36 \\
\hline AN & 41.3 & 100 & - & 33 & I \\
\hline $\mathrm{R}$ & 43.8 & 91.5 & - & 32 & 7 \\
\hline AK & 44.8 & 91.6 & - & 31 & 6 \\
\hline $\mathrm{AU}$ & 58.7 & 98.1 & - & 30 & 2 \\
\hline $\mathrm{N}$ & 59.3 & 65.3 & - & 29 & 27 \\
\hline $\mathrm{AL}$ & 59.8 & 87 & - & 28 & II \\
\hline $\mathrm{AJ}$ & 61.2 & 79.4 & - & 27 & 16 \\
\hline$A D$ & 63.6 & 84.5 & - & 26 & 12 \\
\hline$A C$ & 63.6 & 100 & - & 25 & I \\
\hline$M$ & 63.8 & 80.6 & - & 24 & 14 \\
\hline $\mathrm{AO}$ & 64.3 & 91.1 & - & 23 & 9 \\
\hline$C$ & 66.8 & 69 & - & 22 & 22 \\
\hline$E$ & 69.4 & 80.3 & - & 21 & 15 \\
\hline$A B$ & 72.7 & 92.6 & - & 20 & 5 \\
\hline AM & 76.3 & 67.8 & + & 19 & 23 \\
\hline $\mathrm{AP}$ & 76.4 & 69.1 & + & 18 & 21 \\
\hline $\mathrm{U}$ & 76.6 & 100 & - & 17 & I \\
\hline $\mathrm{K}$ & 77.3 & 75.5 & + & 16 & 17 \\
\hline$A$ & 78 & 93.5 & - & 15 & 4 \\
\hline AA & 78.5 & 81.3 & - & 14 & 13 \\
\hline$B$ & 82.8 & 89.8 & - & 13 & 10 \\
\hline AT & 83.2 & 66.9 & + & 12 & 25 \\
\hline $\mathrm{AF}$ & 83.9 & 100 & - & 11 & 1 \\
\hline$X$ & 90 & 53.7 & + & 10 & 30 \\
\hline 0 & 91.3 & 91.5 & - & 9 & 7 \\
\hline AS & 93.3 & $<30$ & + & 8 & 36 \\
\hline$S$ & 93.8 & 74.3 & + & 7 & 19 \\
\hline$Y$ & 94.9 & 67.8 & + & 6 & 24 \\
\hline Z & 97.2 & 43.2 & + & 5 & 34 \\
\hline D & 97.4 & 74.1 & + & 4 & 20 \\
\hline $\mathrm{V}$ & 98.1 & 55.5 & + & 3 & 29 \\
\hline AG & 98.2 & 58.9 & + & 2 & 28 \\
\hline $\mathrm{AE}$ & 100 & 100 & $=$ & 1 & I \\
\hline $\mathrm{AQ}$ & 100 & 50.4 & + & I & 32 \\
\hline $\mathrm{F}$ & 100 & $<30$ & + & 1 & 36 \\
\hline I & 100 & 100 & $=$ & I & I \\
\hline$P$ & 100 & 36.3 & + & 1 & 35 \\
\hline $\mathrm{T}$ & 100 & 91.5 & + & I & 8 \\
\hline$W$ & 100 & 75.3 & + & I & 18 \\
\hline unida & $7(15 \%)$ & $7(19 \%)$ & -- & -- & -- \\
\hline
\end{tabular}

* Equivalencias: -, puntuación de provisión de servicios menor al de resultados en salud; +, puntuación de provisión de servicios mayor al de resultados en salud; =, puntuación de provisión de servicios igual al de resultados en salud 


\section{Cuadro III}

Magnitud y TIPO de MejoRía POTENCIAL DE LAS UNIDAdES DE SALUD DEL PRIMER NIVEL DE ATENCIÓN QUE OPERA CON MENOS DE 50\% DE EFICIENCIA EN PROVISIÓN DE SERVICIOS Y RESULTADOS DE SALUd. NueVo LeÓN, 2004

\begin{tabular}{ccccc}
\multicolumn{4}{c}{ Potencial de mejoría* en provisión de servicios } \\
\hline \multirow{4}{*}{$\begin{array}{c}\text { Examen } \\
\text { Unual }\end{array}$} & Examen & Medición presión & Medición \\
& anual & arterial en & peso en \\
& pies & fondo de ojo & cada visita & cada visita \\
$\%$ & $\%$ & $\%$ & $\%$
\end{tabular}

\begin{tabular}{lrrrr} 
AK & 100 & 0 & 0 & 0 \\
\hline$R$ & 100 & 40 & 0 & 0 \\
\hline AN & 100 & 0 & 0 & 0 \\
\hline$J$ & 100 & 100 & 54 & 0 \\
\hline L & 100 & 100 & 0 & 0 \\
\hline$G$ & 100 & 100 & 14 & 0 \\
\hline Al & 100 & 22 & 16 & 16 \\
\hline$H$ & 100 & 0 & 0 & 13 \\
\hline AR & 100 & 0 & 14 & 26 \\
\hline AH & 100 & 100 & 14 & 25 \\
\hline Q & 100 & 100 & 0 & 0
\end{tabular}

Potencial de mejoría* en resultados de salud

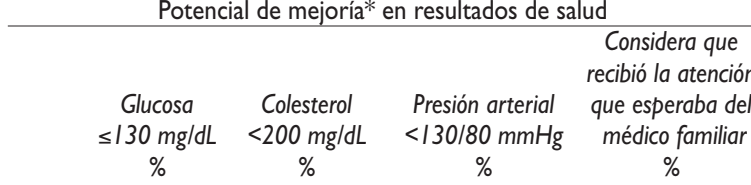

\begin{tabular}{lrrrr} 
AH & 100 & 0 & 0 & 0 \\
\hline$Z$ & 100 & 100 & 100 & 100 \\
\hline$P$ & 100 & 100 & 100 & 100 \\
\hline AS & 100 & 100 & 0 & 0 \\
\hline$J$ & 100 & 0 & 0 & 100 \\
\hline$F$ & 100 & 100 & 100 & 36
\end{tabular}

* Cuanto más alto sea el porcentaje, mayor es el potencial de mejoría en la producción dados los recursos humanos con los que cuenta la unidad de salud

Christensen y colaboradores ${ }^{16}$ analizaron la eficiencia de cinco equipos de salud de un centro de diabetes; el menos eficiente registró una puntuación de 89\%. Baily y Garber ${ }^{17}$ compararon la eficiencia de diabetes de los sectores de salud estadounidense e inglés; Inglaterra registró una puntuación de 100\% y Estados Unidos de $52 \%$. La comparación de puntuaciones de eficiencia entre estudios debe realizarse con precaución porque se refiere a las unidades de salud incluidas en el análisis. En realidad, el AED constituye una herramienta poderosa de benchmarking. Una inspección detallada reveló que las unidades más ineficientes podían incrementar la cantidad de pacientes con revisión de fondo de ojo y pies. La evidencia señala que lo primero disminuye 60 a 70\% la pérdida grave de la visión y lo segundo 50 a $60 \%$ el pie diabético. ${ }^{26}$

Se considera que es factible realizar el estudio de los pies en todos los pacientes, ya que no requiere mayor tecnología, sólo capacitación. Las razones posibles de incumplimiento son la falta de conocimiento sobre su trascendencia, no sentirse con la destreza necesaria, o bien el descuido. Asimismo, debe mejorarse el porcentaje de pacientes con control apropiado de glucosa en ayuno a través de medidas reconocidas como efectivas. ${ }^{26}$ En este sentido, se debe enfatizar en programas de educación médica continua que refuercen su importancia y beneficio. Once unidades registraron puntuaciones de eficiencia de servicios inferiores a 50\% y siete en salud. Estos hallazgos podrían atribuirse al entorno no competitivo en el cual las unidades funcionan a pesar de la extrema ineficiencia. Un principio económico postula que una estructura de mercado competitiva hace que los proveedores busquen la eficiencia, mientras que la falta de incentivos debilita a las organizaciones públicas y privadas no lucrativas. ${ }^{13,14}$ Vale la pena destacar que las unidades ineficientes observaron rendimientos crecientes a escala, lo cual significa que, cuando es posible incrementar el insumo, la producción aumenta a un ritmo mayor que el proporcional a dicho incremento.

Si bien es cierto que la muestra incluyó sólo centros del mismo sector de seguridad social con marco regulador similar, los factores localización de la unidad y total de consultas originaron variación en el nivel de eficiencia de provisión de servicios, no así de resultados en salud. Pai y colaboradores ${ }^{10}$ notificaron discrepancias urbanasrurales por el uso de procedimientos de laboratorio. En cuanto al volumen o el tamaño, las publicaciones no ofrecen resultados concluyentes. Algunos autores reconocen un vínculo positivo con la eficiencia, ${ }^{11,15,27}$ mientras que otros uno negativo. ${ }^{28}$ De igual manera, se observaron diferencias entre eficiencia de servicios y resultados de salud atribuibles a las características del médico; la primera no se vio afectada, pero la segunda sí favorecida por la edad, al margen de los atributos del paciente, quizá por falta de aptitud clínica en los médicos jóvenes ya que se esperaría adquirirla con los años. Se pensaba que un menor tiempo de días laborados podría afectar la eficiencia, dado que si el titular se ausenta, a éste lo reemplaza un médico eventual, casi siempre más joven, con menor antigüedad laboral y experiencia. Sin embargo, no fue así. De forma adicional, la eficiencia puede ser un reflejo del perfil del paciente. Por ejemplo, Chilingerian ${ }^{11}$ señaló que la eficiencia se atenuaba cuanto mayor fuera la edad del enfermo. En esta categoría, el comportamiento fue mixto; así, edad y tiempo con el diagnóstico favorecieron la eficiencia de los servicios, pero perjudicaron la de los resultados en salud. Conviene 


\section{Cuadro IV}

ANÁlisis de REGRESIÓN PARA EXPLICAR LAS FUENTES DE VARIACIÓN DE EFICIENCIA EN LA PROVISIÓN DE SERVICIOS Y RESUltados EN SALUd EN UNIDADES De PRIMER NIVEL de ATENCIÓN. NueVo LeÓN, 2004

\begin{tabular}{|c|c|c|c|c|c|}
\hline \multicolumn{3}{|l|}{ Modelo I } & \multicolumn{3}{|l|}{ Modelo 2} \\
\hline Variable & $\begin{array}{c}\text { Provisión } \\
\text { de servicios } \\
\text { Coeficiente }\end{array}$ & $\begin{array}{l}\text { Resultados } \\
\text { en salud } \\
\text { Coeficiente }\end{array}$ & Variable & $\begin{array}{c}\text { Provisión } \\
\text { de servicios } \\
\text { Coficiente }\end{array}$ & $\begin{array}{l}\text { Resultados } \\
\text { en salud } \\
\text { Coeficiente }\end{array}$ \\
\hline Ubicación de la unidad, zona metropolitana & $48.60 *$ & -7.63 & Total de consultas & $0.0002 *$ & -0.00009 \\
\hline Edad del médico & 0.39 & $1.50 *$ & Edad del médico & 0.35 & $1.86 *$ \\
\hline Días laborados del médico & $0.09 \ddagger$ & 0.007 & Días laborados del médico & 0.05 & 0.02 \\
\hline Edad del paciente & 0.37 & $-0.44^{\ddagger}$ & Edad del paciente & $0.65^{*}$ & $-1.23 *$ \\
\hline Escolaridad del paciente hasta la primaria & $0.66^{*}$ & -0.17 & Escolaridad del paciente hasta la primaria & 0.13 & -0.2 \\
\hline Tiempo con el diagnóstico & $3.64 *$ & $-2.70 *$ & Tiempo con el diagnóstico & $3.47^{*}$ & -2 \\
\hline Tiempo de afiliación & $-1.26 *$ & 0.7 & Tiempo de afiliación & $-0.99 \ddagger$ & $0.88^{\ddagger}$ \\
\hline Promedio de visitas de control & 0.87 & $2.90 *$ & Promedio de visitas de control & 0.19 & $2.68^{*}$ \\
\hline Constante & $-178.6 \ddagger$ & $-67.9 *$ & Constante & $-134.3 *$ & $-42.7^{*}$ \\
\hline Log Likelihood & 191 & 190 & Log Likelihood & 196 & 189 \\
\hline $\mathrm{R} 2$ & $0.5 I^{*}$ & $0.11 \ddagger$ & $\mathrm{R} 2$ & $0.3 I^{*}$ & $0.13^{\ddagger}$ \\
\hline $\begin{array}{l}* p \leq .01 \\
\ddagger p \leq .05\end{array}$ & & & & & \\
\hline
\end{tabular}

R2= cuadrado del coeficiente de correlación $r$ entre los valores esperados y los observados en la bondad de ajuste

hacer referencia a la variación de eficiencia explicada por los modelos utilizados, no más de $51 \%$ para provisión de servicios y de $13 \%$ para la salud. Los indicadores de resultado incluidos en el estudio reflejan de manera indirecta el proceso terapéutico, en el cual además intervienen factores no analizados, como la calidad de la relación médico-paciente y los estilos propios de la práctica médica. Asimismo, el autocuidado por parte del paciente puede propiciar pobres resultados..$^{29}$ Por consiguiente, ciertos factores adicionales podrían mejorar el poder explicativo de modelos similares y completar las conclusiones inferidas de este trabajo.

Limitaciones del estudio. Los insumos y los productos se obtuvieron sólo una vez y, por lo tanto, no fue posible analizar la eficiencia en el transcurso del tiempo, pero esto se considerará en un futuro cercano. No debe olvidarse que los resultados obtenidos provienen de datos agregados tras utilizar como unidad de análisis la clínica de medicina familiar, lo que permite hacer deducciones sólidas a nivel organizacional, pero no a nivel individual del médico, por lo que es necesario proseguir el trabajo en esta línea de investigación. Cabe mencionar que en ningún momento se pretendió vincular la eficiencia de provisión de servicios con la de resultados en salud. La primera incluyó tan sólo indicadores de atención al paciente para detección temprana de complicaciones y no datos inherentes al tratamiento $\mathrm{u}$ otros factores más íntimamente relacionados con los indicadores de salud empleados en este protocolo. Se debe tener precaución al correlacionar una dimensión con otra y considerar a la primera como variable explicativa de la segunda.

Para concluir, los resultados expuestos señalaron que hay unidades que son eficientes para otorgar servicios, en tanto que otras se enfocan en el control de la enfermedad y expectativas del paciente; empero, muy pocas clínicas lograron ambos productos, por lo que se requiere diferenciar su medición. Vale la pena destacar que en México, esta información constituye un primer esfuerzo de estimación de eficiencia en provisión de servicios y resultados en salud del cuidado al paciente con diabetes en instalaciones de atención primaria de una institución del sector público. Debido a que la eficiencia de las unidades de medicina familiar puede relacionarse con factores organizacionales y atributos del paciente, es importante identificarlos para adoptar las medidas correspondientes. La eficiencia representa un reto especial para las organizaciones de salud no lucrativas, como la presente; ésta, como todas, está interesada en otorgar servicios eficientes sin sacrificar la calidad con recursos limitados. En realidad, la importancia de los estudios de eficiencia radica en ofrecer a las instituciones de salud el camino por el cual se puede empezar a optimizar. 


\section{Agradecimientos}

Los autores están en deuda con todo el personal de salud que facilitó el trabajo de campo en las unidades de medicina familiar, en particular con los directores médicos. Asimismo, agradecen el apoyo de CONACYT, sin cuyo apoyo financiero no habría sido posible realizar el presente trabajo de investigación (No. 2002-CO1-6852).

\section{Referencias}

I. Puentes E, Gómez-Dantés O, Martínez T. Salud: México 200|•2005. Información para la rendición de cuentas. México, DF: Secretaría de Salud, 2006:7.

2. Información Estadística en Salud del Instituto Mexicano del Seguro Social. [Consultado en septiembre 18,2007$]$. Disponible en: www.imss. gob.mx.

3. Sistema Nacional de Información en Salud de la Secretaría de Salud. [Consultado en septiembre 18, 2007]. Disponible en: http://sinais.salud. gob.mx.

4.American Diabetes Association. Economic costs of diabetes in the U.S. in 2007. Diab Care 2008:31:596-615.

5. Economic and Health Costs of Diabetes: HCUP Highlight Issue I. AHRQ Publication No. 05-0034, January 2005. Agency for Healthcare Research and Quality. [Consultado en octubre 8, 2007]. Disponible en: http://www.ahrq.gov/data/hcup/highlightl/highl.htm.

6. Allen J, Gay B, Crebolder H, Heyrman J, Svab I, Ram P.The european definitions of the key features of the discipline of general practice: the role of the GP and core competencies. Br J Gen Pract 2002;52:526-527. 7. Puig-Junoy J. Eficiencia en la atención primaria de salud: Una revisión crítica de las medidas de frontera. Rev Esp Salud Pública 2000;74:483-495 8. Palmer S, Torgerson DJ. Economic notes: definitions of efficiency. BMJ 1999;318:1136

9. Hussain A, Brightman JR. White paper. Frontier analyst in depth. UK: Banxia Software LTD, 2000.

10. Pai CW, Ozcan YA, Jiang HJ. Regional variation in physician practice pattern: an examination of technical and cost efficiency for treating sinusitis. J Med Syst 2000; 24:103-1 17.

II. Chilingerian JA. Evaluating physician efficiency in hospitals: a multivariate analysis of best practices. Eur J Oper Res 1995;80:548-574. 12.Akinci F, Campbell CR,Association for Health Services Research. Meeting. Technical efficiency in federally-funded community health center (CHCs): the role of managed care. Abstr Book Assoc Health Serv Res Meet 1999;16:247.

13. Rosko MD, Chilingerian JA, Zinn JS,Aaronson WE. The effects of ownership, operating environment, and strategic choices on nursing home efficiency. Med Care 1995;33:100I-1021.

14. Wheeler JR, Fadel H, D'Aunno TA. Ownership and performance of outpatient substance abuse treatment centers. Am J Public Health 1992;82:7|I-7|8.

15. Chang H. Determinants of hospital efficiency: the case of central government-owned hospitals in Taiwan. Omega 1998;26:307-317.
16. Christensen MC, Binder C, Hansen JB, Lyholm, B. Frontier practice in diabetes care: a method for estimating relative efficiency in diabetes care. 2003 (abstract Number: 2245-PO). [Consultado en junio 26, 2006.]Disponible en: http://scientificsessions. diabtes.org/ abstracts. 17. Baily MN, Garber AM. Health care productivity. In: Baily MN, Reiss PC, Winston C (ed.). Brookings Papers on Economic Activity. Microecnomics 1997. Washington: Brookings Institution Press, 1998:143-202.

18. Chilingerian JA, Sherman HD. Health Care Applications: from hospitals to physicians, from productive efficiency to quality frontiers. In: Cooper WW, Seiford LM, Zhu J (ed.). Handbook of data envelopment analysis. Hingham: Kluwer Academic Publishers, 2004: 48I-537.

19. Cooper WW, Seiford LM, Zhu J. Data envelopment analysis. History, models and interpretations. In: Cooper WW, Seiford LM, Zhu J (ed.). Handbook on data envelopment analysis. Boston: Kluwer Academic Publishers, 2004: I-40.

20. Consenso para la prevención de las complicaciones crónicas de la diabetes tipo 2. Rev Inves Clin 2000;52:325-363.

21.American Diabetes Association. Standards of medical care in diabetes. Diab Care 2005;(Suppl I):S4-S36

22. Oláiz G, Rojas R, Barquera S, Shamah T, Aguilar C, Cravioto P, et al. Encuesta Nacional de Salud 2000. Tomo 2. La salud de los adultos. Cuernavaca: Instituto Nacional de Salud Pública, 2003:94.

23. Bleda-Hernández MJ,Tobías-Garcés A. Aplicación de los modelos de regresión Tobit en la modelización de variables epidemiológicas censuradas. Gac Sanit 2002;16:188-195.

24. UCLA Academic Technology Services. Stata Data Analysis Examples. Tobit Analysis. [Consultado en octubre 8, 2007]. Disponible en: http:// www.ats.ucla.edu/stat/stata/dae/tobit.htm.

25. Bierens HJ. EasyReg International. Pennsylvania: Pennsylvania State University, 2006.

26. Narayan KM, Gregg EW, Engelgau MM, Moore B, Thompson T], Williamson DF, et al. Translation research for chronic disease: the case of diabetes. Diab Care 2000;23:1794-1798.

27. Peñaloza-Ramos MC. Evaluación de la eficiencia en instituciones hospitalarias públicas y privadas con Data Envelopment Analysis (DEA). Archivos de Economía República de Colombia. Departamento Nacional de Planeación. Dirección de Estudios Económicos. Documento 244, 2003. Disponible en: http://www.dnp.gov.co/PortalWeb/Portals/0/archivos/ documentos/DEE/Archivos_Economia/244.pdf

28. Pinzón-Martínez MJ. Medición de eficiencia técnica relativa en hospitales públicos de baja complejidad mediante la metodología Data Envelopment Analysis (DEA).Archivos de Economía República de Colombia. Departamento Nacional de Planeación. Dirección de Estudios Económicos. Documento 245, 2003. Disponible en: http://www.dnp.gov.co/PortalWeb/ Portals/0/archivos/documentos/DEE/Archivos_Economia/245.pdf 29. Harris MI. Health care and health status and outcomes for patients with type 2 diabetes. Diab Care 2000;23:754-758.

30. Dirección General de Evaluación del Desempeño de la Secretaría de Salud. [Consultado en septiembre 19,2007]. Disponible en: http:// evaluacion.salud.gob.mx.

31. Instituto Nacional de Estadística, Geografía e Informática. Información estadística. [Consultado en septiembre 19, 2007]. Disponible en: www. inegi.gob.mx. 
Anexo I

Perfil de las unidades de salud incluidas en el estudio. Nuevo León, 2004

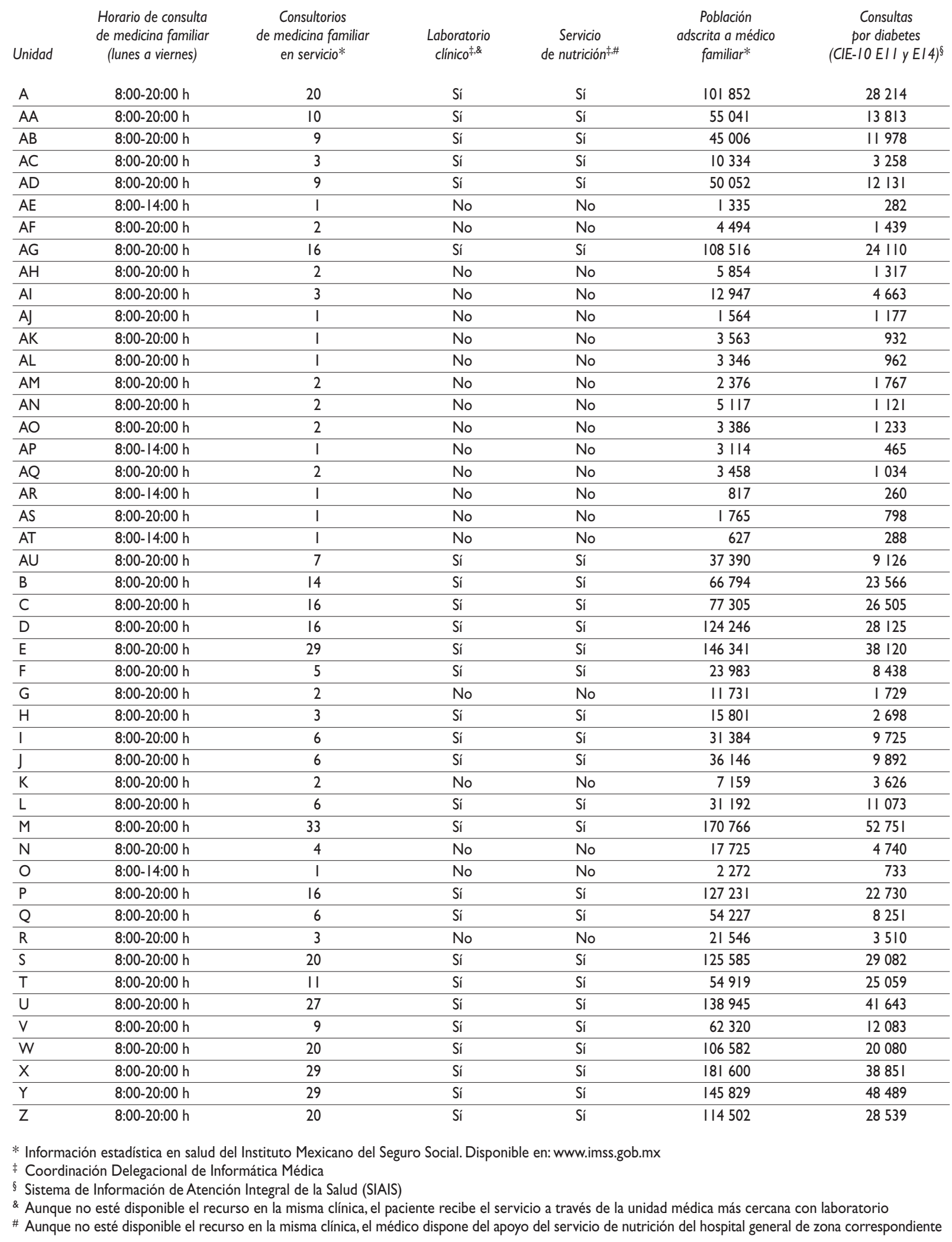


Anexo 2

Perfil de los pacientes incluidos en el estudio. Nuevo León, 2004

\begin{tabular}{|c|c|c|c|c|c|c|}
\hline & & & Sexo femenino & Hasta primaria & Tiempo c & (años) \\
\hline & Media & $D E$ & $\%$ & $\%$ & Media & $D E$ \\
\hline A & 61.2 & 12.7 & 62.2 & 68.9 & 11.4 & 9.6 \\
\hline AA & 58.9 & 12 & 71.4 & 76.2 & II.I & 12.6 \\
\hline$A B$ & 66 & 11.7 & 66.7 & 55.6 & 14.3 & 9.7 \\
\hline$\overline{A C}$ & 63.3 & 8.1 & 66.7 & 55.6 & 10.6 & 7.9 \\
\hline$\overline{A D}$ & 55.2 & 9.1 & 88.9 & 83.3 & 7.2 & 6.7 \\
\hline$\overline{\mathrm{AE}}$ & 55.4 & 12.4 & 77.8 & 77.8 & 9.8 & 7.7 \\
\hline $\mathrm{AF}$ & 56.7 & 12.9 & 66.7 & 77.8 & 9.3 & 8.9 \\
\hline AG & 53.5 & 12 & 81.3 & 62.5 & 7.2 & 7.2 \\
\hline $\mathrm{AH}$ & 56.6 & 9.3 & 60 & 80 & 8.8 & 4 \\
\hline $\mathrm{Al}$ & 57.1 & 15.5 & 60 & 50 & 6.3 & 4.3 \\
\hline AJ & 60.5 & 11.5 & 63.6 & 90.9 & 9.9 & 7.2 \\
\hline AK & 69.6 & 9.4 & 50 & 87.5 & 14.3 & 7.3 \\
\hline$\overline{\mathrm{AL}}$ & 55.2 & 9.2 & 45.5 & 90.9 & 13.4 & 7.9 \\
\hline$\overline{A M}$ & 58 & 11.7 & 70 & 80 & II.I & 8.9 \\
\hline AN & 64 & 10.8 & 50 & 100 & 8.8 & 7.1 \\
\hline $\mathrm{AO}$ & 60.1 & 15.8 & 57.1 & 85.7 & 9.9 & 10.7 \\
\hline $\mathrm{AP}$ & 59.4 & 13 & 62.5 & 100 & 10.8 & 6.6 \\
\hline $\mathrm{AQ}$ & 59.4 & 5 & 60 & 90.0 & 13.7 & 7 \\
\hline AR & 56.7 & 6.2 & 55.6 & 66.7 & 7.1 & 5 \\
\hline AS & 60.2 & 5.8 & 66.7 & 100 & 8.3 & 7.9 \\
\hline AT & 60.3 & 8.1 & 100 & 88.9 & 13.5 & 6.4 \\
\hline $\mathrm{AU}$ & 54.6 & 5.3 & 77.8 & 44.4 & 5.1 & 4.1 \\
\hline$B$ & 63.3 & 10.9 & 62.7 & 62.7 & 10.2 & 9.6 \\
\hline $\mathrm{C}$ & 63.8 & 9.4 & 85 & 70 & 11.7 & 9.5 \\
\hline$D$ & 53.9 & 8.9 & 93.3 & 73.3 & 11.1 & 10.5 \\
\hline$E$ & 56.1 & 9.2 & 67.9 & 75 & 9.4 & 8.7 \\
\hline $\mathrm{F}$ & 64.3 & 11.1 & 100 & 71.4 & 16.6 & 14 \\
\hline $\mathrm{G}$ & 59.8 & 11.1 & 83.3 & 100 & 8.5 & 5.6 \\
\hline $\mathrm{H}$ & 55.5 & 8.9 & 100 & 80 & 7.9 & 3.2 \\
\hline 1 & 68.6 & 9.3 & 62.5 & 100 & 10.6 & 9.5 \\
\hline $\mathrm{J}$ & 61.8 & 11.3 & 60 & 88.9 & 11.4 & 7 \\
\hline $\mathrm{K}$ & 60.6 & 11.1 & 45.5 & 100 & 8.9 & 6.9 \\
\hline $\mathrm{L}$ & 57.7 & 10.4 & 50 & 80 & 9.7 & 3.8 \\
\hline$M$ & 59.8 & 9.2 & 84.1 & 68.3 & 11.1 & 7.2 \\
\hline $\mathrm{N}$ & 64 & 1.1 & 66.7 & 100 & 6 & 2 \\
\hline 0 & 59.9 & 9.2 & 90 & 90 & 11.1 & 7.7 \\
\hline$P$ & 56.7 & 11.8 & 80 & 86.7 & 11.1 & 8.5 \\
\hline $\mathrm{Q}$ & 55.7 & 13.9 & 42.9 & 85.7 & 4.1 & 2.9 \\
\hline$R$ & 57.3 & 12.8 & 70 & 90 & 14.6 & 9.7 \\
\hline$S$ & 58.5 & 11.9 & 80 & 68.6 & 9.6 & 6.7 \\
\hline$T$ & 58.5 & 9.2 & 53.1 & 71.9 & 6.3 & 5.6 \\
\hline$U$ & 61.8 & 10.9 & 67.7 & 75.4 & 10.2 & 9.6 \\
\hline $\mathrm{V}$ & 58.9 & 9.6 & 89.5 & 84.2 & 7.9 & 6.1 \\
\hline$W$ & 58.1 & 11.9 & 86 & 80.7 & 9.1 & 6.5 \\
\hline$x$ & 57.9 & 10 & 83.3 & 74.2 & 8.6 & 8.1 \\
\hline $\bar{Y}$ & 57.7 & 10.3 & 79.5 & 69 & 8.7 & 8.7 \\
\hline $\bar{Z}$ & 59.8 & II.I & 63.2 & 84.2 & 12.1 & 9.8 \\
\hline
\end{tabular}

